\title{
Highly Coherent Femtosecond Electron Pulses for Ultrafast Transmission Electron Microscopy
}

\author{
Nora Bach $^{1 *}$, Armin Feist ${ }^{1}$, Till Domröse ${ }^{1}$, Thomas Danz ${ }^{1}$, Marcel Möller ${ }^{1}$, Nara Rubiano \\ da Silva ${ }^{1}$, Katharina Priebe ${ }^{1}$, Christopher Rathje ${ }^{2}$, Sascha Schäfer ${ }^{1,2}$, and Claus Ropers ${ }^{1}$ \\ ${ }^{1}$ IV. Physical Institute - Solids and Nanostructures, University of Göttingen, Friedrich-Hund-Platz 1, \\ D-37077 Göttingen, Germany \\ ${ }^{2}$ Institute of Physics, University of Oldenburg, Carl-von-Ossietzky-Straße 9-11, 26129 Oldenburg, \\ Germany
}

\begin{abstract}
We describe the implementation and detailed characterization of a laser-triggered field-emitter electron source integrated into a modified transmission electron microscope. Highly coherent electron pulses enable high resolution ultrafast electron imaging and diffraction.
\end{abstract}

\section{Introduction}

In recent years, ultrafast physics has revealed a detailed and rich picture of the fundamental interactions between the electron, spin and lattice subsystems in spatially homogeneous condensed matter. Ultrafast dynamics in strongly inhomogeneous and nanostructured systems require novel experimental techniques combining an ultrafast temporal with a nanoscale spatial resolution. Ultrafast Transmission Electron Microscopy (UTEM) is a particularly promising approach, in which femtosecond electron pulses are employed to stroboscopically probe ultrafast nanoscale dynamics in a laser-pump/electron-probe scheme (Fig. 1a). Whereas conventional transmission electron microscopy routinely achieves atomic-scale spatial resolution, imaging in UTEM is typically limited by the coherence properties of ultrashort electron pulses. In particular, the micrometer-scale electron source size of flat photocathodes only supports a limited transverse coherence length, rendering UTEM experiments on a few-nanometer length scale challenging. Here, we report on the linear photoemission from needle-shaped photocathodes for which the emission is confined to nanometer sized areas, resulting in highly-coherent electron pulses [3-6]. Additionally, we illustrate that we can use the advanced beam properties to conduct ultrafast convergent beam electron diffraction experiments, perform Lorentz magnetic imaging and record highresolution electron energy loss spectra.

\footnotetext{
* Corresponding author: nora.bach@uni-goettingen.de
} 


\section{Development and Characterization of Laser-Triggered Field Emitter}

In the Göttingen UTEM, we generate electron pulses by single-photon photoemission from a laser-driven Schottky field-emitter. The emitter is based on a single-crystalline (100)oriented tungsten needle, which is covered with a zirconium oxide overlayer. In general, Schottky emitters are known as high-brightness continuous electron sources, and are usually operated at an elevated temperature of about $1800 \mathrm{~K}$ and an applied electric field in the range of $0.5-1 \mathrm{~V} / \mathrm{nm}$ [7]. For a (100)-oriented apex facet, the $\mathrm{ZrO}$ overlayer results in a selective workfunction reduction, supporting tunneling-assisted thermal electron emission at moderate temperatures. For their use as photocathodes, the facet-specific reduction of the work function can be utilized to trigger localized linear photoemission from the tip apex. Specifically, we operate the photocathode at a decreased temperature for which the continuous thermal emission vanishes, and illuminate the apex with femtosecond laser pulses at a central wavelength of $400 \mathrm{~nm}$ (Fig. 1b). A linear scaling of the photoelectron current with the incident optical fluence is observed, allowing for a flexible tuning of the temporal electron pulse structure. The photoelectron pulses are accelerated to $120 \mathrm{keV}$ and coupled into a transmission electron microscope column. In the space-charge-free regime, the electron pulses show a narrow spectral width of $0.6 \mathrm{eV}$ and a pulse duration of $200 \mathrm{fs}$ (full-width-at-half-maximum, characterized by electron light interferometry [8]) (Fig. 1e, f). In order to quantitatively characterize the beam brightness and emittance, caustic scans have been performed for different excitations of the TEM condenser lens system. Advanced beam properties of probe sizes below $1 \mathrm{~nm}$ (Fig. 1c), a beam emittance down to $1.8 \mathrm{~nm} \mathrm{mrad}$ and a degree of transverse coherence exceeding $10 \%$ have been demonstrated using the interference fringes generated by a Möllenstedt biprism provided by the Lichte group in Dresden (Fig. 1d) [8].
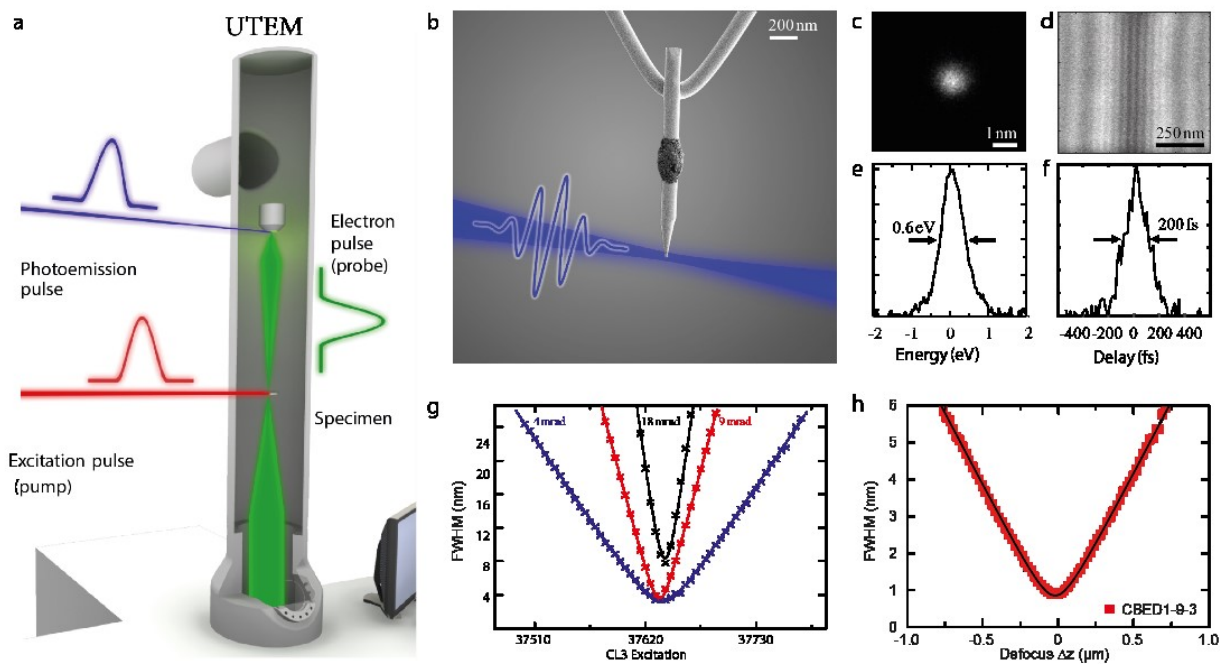

Fig. 1. (a) Schematic of the Göttingen UTEM with stroboscopic laser-pump/electron probe principle. (b) Photoelectron pulses are generated by side illumination of a single-crystalline, (100)-oriented, facetted tungsten tip emitter attached to a heating filament with a $\mathrm{ZrO}_{2}$ reservoir deposited on the emitter shank. (c,e,f) Currently achievable electron focal spot size, and the spectral and temporal width in a space-charge-free regime. (d) Exemplary photoelectron hologram generated with a Möllenstedt biprism. (Figure adapted from Ref. [8].) 
Besides the new opportunities for coherent manipulation of the electron quantum state, ultimately leading to the compression of electron pulse trains into attosecond bursts [9], UTEM is a unique tool to study structural, electronic, as well as magnetic dynamics on the nm-fs scale. In first experiments (Fig. 2), we demonstrated the broad applicability of ultrashort electron pulses in high-resolution imaging and diffraction, Lorentz microscopy, electron energy loss spectroscopy and convergent beam electron diffraction [10], yielding access to ultrafast nanoscale dynamics in multiple degrees of freedom.
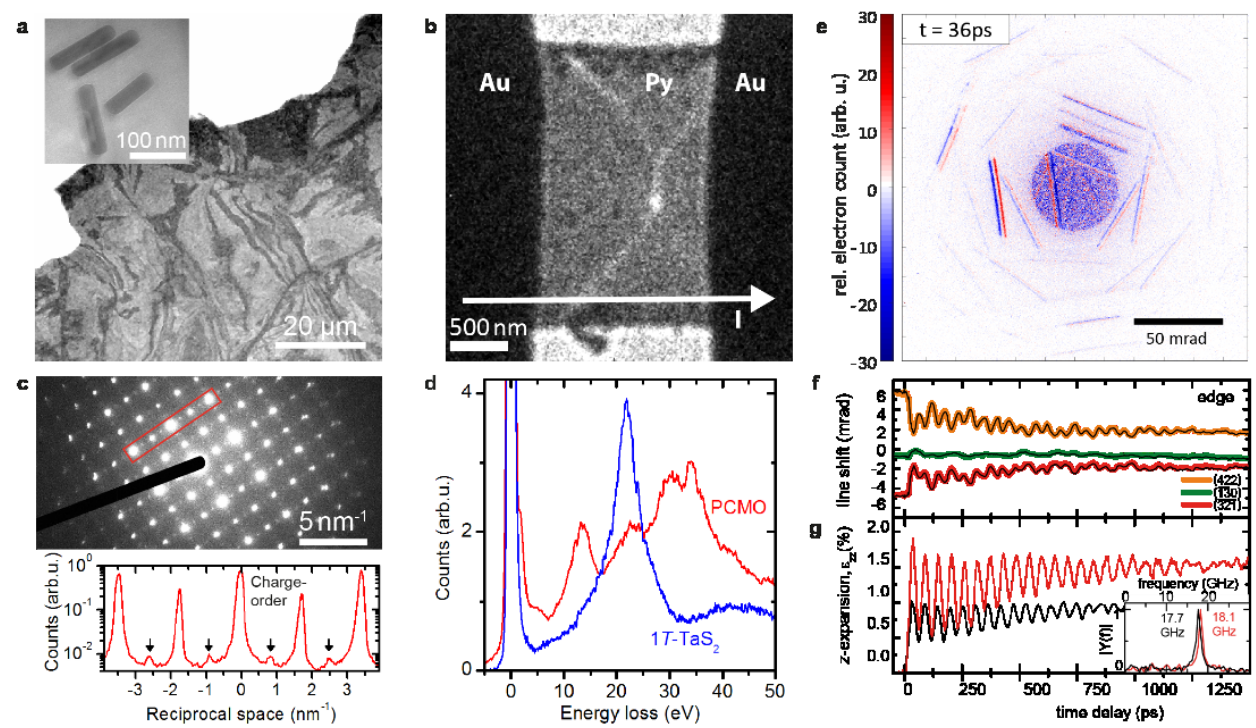

Fig. 1. Exemplary applications of the UTEM instrument. (a) Bright-field image of an ultramicrotomed $50 \mathrm{~nm}$ thin sample of $1 \mathrm{~T}-\mathrm{TaS}_{2}$, showing bending contrast of the thin-film membrane and drop-cast gold particles on the sample surface. (b) Lorentz imaging of the magnetic domain structure in a $20 \mathrm{~nm}$ thick permalloy square contacted by two gold electrodes. (c) Diffraction pattern of the charge-ordered phase of ion-polished PCMO. (d) Electron energy loss spectra of 1T-TaS2 and PCMO. (e) Time-dependent Bragg-line changes of a single-crystalline graphite flake. (f) Experimentally obtained delay-dependent center-of-mass shift (black line) and reconstructed mean line position (colored line) of the (422), (-1-30) and (-3-2-1) Bragg lines, probed at the graphite edge. (g) Reconstructed z-axis expansion with Fourier analysis (inset, $|\mathrm{Y}(\mathrm{f})|$ : Fourier amplitude) (Figure adapted from Ref. $[8,10])$.

\section{References}

1. H. Dömer, Rev. Sci. Instrum. 74, 4369 (2003)

2. A. H. Zewail, Science 328, 187 (2010)

3. P. Hommelhoff, Phys. Rev. Lett. 97, 24740 (2006)

4. C. Ropers, Phys. Rev. Lett. 98, 043907 (2007)

5. A. Paarmann, J. Appl. Phys. 112, 113109 (2012)

6. B. Cook, Ultramicroscopy 109, 403 (2009)

7. M. Fransen, Appl. Surf. Sci. 146, 357 (1999)

8. A. Feist, Ultramicroscopy 176, 63 (2017)

9. K. E. Priebe, Nature Phot. 11, 793 (2017)

10. A. Feist, Struct. Dyn. 5, 14302 (2018) 